PROCEEDINGS OF THE

AMERICAN MATHEMATICAL SOCIETY

Volume 128, Number 5, Pages 1293-1299

S 0002-9939(99)05184-

Article electronically published on August 5, 1999

\title{
ON CASTELNUOVO-MUMFORD REGULARITY OF PROJECTIVE CURVES
}

\author{
ISABEL BERMEJO AND PHILIPPE GIMENEZ
}

(Communicated by Wolmer V. Vasconcelos)

\begin{abstract}
We give an effective method to compute the regularity of a saturated ideal $I$ defining a projective curve that also determines in which step of a minimal graded free resolution of $I$ the regularity is attained.
\end{abstract}

\section{INTRODUCTION}

Let $S:=K\left[x_{0}, \ldots, x_{n}\right]$ be a polynomial ring over an algebraically closed field $K$, and let $I$ be a homogeneous ideal of $S$ defining a subscheme $\mathfrak{X}$ of projective $n$ space $\mathbb{P}_{K}^{n}$. The Castelnuovo-Mumford regularity (or simply regularity) of $I$, reg $I$, is defined as follows: if

$$
0 \rightarrow \bigoplus_{j=1}^{\beta_{p}} S\left(-e_{p j}\right) \stackrel{\varphi_{p}}{\longrightarrow} \cdots \stackrel{\varphi_{1}}{\longrightarrow} \bigoplus_{j=1}^{\beta_{0}} S\left(-e_{0 j}\right) \stackrel{\varphi_{0}}{\longrightarrow} I \rightarrow 0
$$

is a minimal graded free resolution of $I$, setting $e_{i}:=\max \left\{e_{i j} ; 1 \leq j \leq \beta_{i}\right\}$, then $\operatorname{reg} I:=\max \left\{e_{i}-i ; 0 \leq i \leq p\right\}$. In other words, reg $I$ is the smallest integer $m$ for which $I$ is $m$-regular, i.e. $e_{i j} \leq m+i$ for all $i, j$ (see [2, Def. 3.2] for equivalent definitions). When $I$ is saturated (i.e. when it is the largest ideal defining $\mathfrak{X}$ ), we call this the regularity of $\mathfrak{X}$ (see 2 , Sect. 1]).

The regularity is a numerical invariant of the ideal $I$ and is, as said in [6], "an important measure of how hard it will be to compute a free resolution". In fact, knowing it beforehand avoids unnecessary computation in large degrees while obtaining the minimal graded free resolution of $I$ through Buchberger's syzygy algorithm (see [3]).

In this paper, we shall essentially be concerned with the regularity of a saturated ideal $I$ defining a subscheme $\mathfrak{X}$ of $\mathbb{P}_{K}^{n}$ of dimension one.

In Section 1, we show a general property of finitely generated graded $S$-modules asserting that the regularity of $M$ is determined by the tail of the minimal graded free resolution (Proposition 1.11). As a consequence we obtain that, in our case, reg $I$ is equal to either $e_{n-1}-n+1$ or $e_{n-2}-n+2$, i.e. the regularity is always attained at one of the last two steps of the resolution.

Received by the editors June 23, 1998.

1991 Mathematics Subject Classification. Primary 13D45; Secondary 14Q05, 13D40.

Key words and phrases. Regularity, projective curves, Hilbert functions.

The first author was supported in part by D.G.U.I., Gobierno de Canarias.

The second author was supported in part by D.G.I.C.Y.T., PB94-1111-C02-01. 
Assuming that $K\left[x_{n-1}, x_{n}\right]$ is a Noether normalization of $S / I$, we give in Section 2 an effective method to compute the regularity of $I$ that does not require the knowledge of a minimal graded free resolution of $I$ (Theorem[2.7). The idea is to introduce an arithmetically Cohen-Macaulay curve whose regularity is closely related with that of $\mathfrak{X}$. For this reason, we first focus on the Cohen-Macaulay case (Theorem 2.4). These two theorems together with an effective criterion to determine whether $\mathfrak{X}$ is arithmetically Cohen-Macaulay (Proposition 2.1), give an algorithm to compute the regularity of $I$. Using Section 11, this algorithm also determines in which step of a minimal graded free resolution of $I$, reg $I$ is attained.

\section{Where is the REgularity attained?}

Let $M$ be a finitely generated graded $S$-module and consider a minimal graded free resolution of $M$ :

$$
0 \rightarrow F_{p} \stackrel{\varphi_{p}}{\longrightarrow} \cdots \stackrel{\varphi_{1}}{\longrightarrow} F_{0} \stackrel{\varphi_{0}}{\longrightarrow} M \rightarrow 0,
$$

with $F_{i}=\bigoplus_{j=1}^{\beta_{i}} S\left(-e_{i j}\right)$. We denote by $e_{i}:=\max \left\{e_{i j} ; 1 \leq j \leq \beta_{i}\right\}$.

Using spectral sequences, Schenzel proved that the regularity of $M$ is determined by the tail of (1.1) ([10, Thm. 3.11]). We propose here a different proof of this issue based on an observation of Herzog relating the vanishing of a row in some matrix in (1.1) and the regularity of $M$ when $M$ is Cohen-Macaulay ([11, Cor. B.4.1]). Our treatment is both elementary and carries some additional information.

Proposition 1.1. Let $M$ be a finitely generated graded $S$-module and let (1.1) be a minimal graded free resolution of $M$. Denoting $c:=n+1-\operatorname{dim} M$, one has:

$$
e_{0}<e_{1}<\cdots<e_{c} .
$$

Proof. Assume the claim is false. Then for some $i, 1 \leq i \leq c$, the matrix $M_{i}$ describing $\varphi_{i}: F_{i} \rightarrow F_{i-1}$ has a zero row.

Consider now the head of the minimal graded free resolution (1.1) of $M$ :

$$
F_{c} \stackrel{\varphi_{c}}{\longrightarrow} F_{c-1} \stackrel{\varphi_{c-1}}{\longrightarrow} \cdots \stackrel{\varphi_{1}}{\longrightarrow} F_{0} \stackrel{\varphi_{0}}{\longrightarrow} M \rightarrow 0
$$

and apply $\operatorname{Hom}_{S}(., S)$ to this complex. Setting $N:=$ Coker $\varphi_{c}^{\star}$, one gets

$$
F_{0}^{\star} \stackrel{\varphi_{1}^{\star}}{\longrightarrow} F_{1}^{\star} \stackrel{\varphi_{2}^{\star}}{\longrightarrow} \cdots \stackrel{\varphi_{c}^{\star}}{\longrightarrow} F_{c}^{\star} \longrightarrow N \rightarrow 0
$$

which is a complex whose homology is $\operatorname{Ext}_{S}^{i}(M, S)=0$ for $i<c$. Thus, (1.2) is the head of a minimal graded free resolution of $N$, contradicting the fact that the matrix describing $\varphi_{i}^{\star}$, the transpose of $M_{i}$, has a zero column.

Consider a homogeneous ideal $I$ of $S$ and a minimal graded free resolution (0.1) of $I$. The following is a direct consequence of the above proposition.

Corollary 1.2. $\operatorname{reg} I=\max \left\{e_{i}-i ; n-\operatorname{dim} S / I \leq i \leq p\right\}$.

\section{How to COMpute the Regularity?}

Let $I$ be a homogeneous ideal of $S$ defining a not necessarily reduced projective curve $\mathfrak{C}$ in $\mathbb{P}_{K}^{n}$. Assume that $K\left[x_{n-1}, x_{n}\right]$ is a Noether normalization of $S / I$ (i.e. $K\left[x_{n-1}, x_{n}\right] \hookrightarrow K\left[x_{0}, \ldots, x_{n}\right] / I$ is an integral ring extension). Monomials in $S$ will 
be denoted by $\mathbf{x}^{\alpha}:=x_{0}^{\alpha_{0}} \cdots x_{n}^{\alpha_{n}}$, with $\alpha=\left(\alpha_{0}, \ldots, \alpha_{n}\right) \in \mathbb{N}^{n+1}$. Let in $(I)$ denote the initial ideal of $I$ with respect to the reverse lexicographic order.

Consider the evaluation morphism $\theta$ (resp. $\chi): K\left[x_{0}, \ldots, x_{n}\right] \rightarrow K\left[x_{0}, \ldots, x_{n-2}\right]$ defined by $x_{n} \mapsto 0$ (resp. $x_{n} \mapsto 1$ ), $x_{n-1} \mapsto 0$ (resp. $x_{n-1} \mapsto 1$ ) and $x_{i} \mapsto x_{i}$ for $i \notin\{n-1, n\}$. Let $\tilde{I}$ be the ideal of $S$ generated by $\chi(\operatorname{in}(I))$. $\tilde{I}$ is a primary monomial ideal such that $\operatorname{in}(I) \subseteq \tilde{I}$ and $\tilde{I}$ defines a projective curve $\tilde{\mathfrak{C}} \subseteq \mathbb{P}_{K}^{n}$ of degree $\operatorname{deg} \tilde{\mathfrak{C}}=\operatorname{deg} \mathfrak{C}$ (see [5, Lemme 1]).

Denote by $I_{0}$ the ideal $I_{0}:=\theta(I) S \subset S$. As in $\left(I_{0}\right)=\theta($ in $(I)) S$, then in $\left(I_{0}\right) \subseteq$ in $(I)$ and so the degree of the curve $\mathfrak{C}_{0} \subseteq \mathbb{P}_{K}^{n}$ defined by $I_{0}$ satisfies $\operatorname{deg} \mathfrak{C}_{0} \geq \operatorname{deg} \mathfrak{C}$.

Define $F:=\left\{\alpha=\left(\alpha_{0}, \ldots, \alpha_{n-2}\right) \in \mathbb{N}^{n-1} \mid \mathbf{x}^{(\alpha, 0,0)} \in \tilde{I}-\right.$ in $\left.\left(I_{0}\right)\right\} \subset \mathbb{N}^{n-1}$. As $K\left[x_{n-1}, x_{n}\right]$ is a Noether normalization of $S / I, F$ is finite (possibly empty). The following is a criterion to determine, in terms of $F$, whether $S / I$ is Cohen-Macaulay (i.e. whether $\mathfrak{C}$ is an arithmetically Cohen-Macaulay projective curve). It implies that $S / I$ is Cohen-Macaulay if and only if $S /$ in $(I)$ is Cohen-Macaulay, and that $S / I_{0}$ and $S / \tilde{I}$ are Cohen-Macaulay.

Proposition 2.1. $S / I$ is Cohen-Macaulay if and only if $F=\emptyset$.

Proof. Observe that $F=\emptyset$ is equivalent to in $\left(I_{0}\right)=\operatorname{in}(I)$. As $S / I$ is CohenMacaulay if and only if $\left\{x_{n-1}, x_{n}\right\}$ is a regular sequence on $S / I$ (9, Ch. 3, Prop. 4.4]), we shall prove that in $\left(I_{0}\right)=$ in $(I)$ if and only if $\left\{x_{n-1}, x_{n}\right\}$ is a regular sequence on $S / I$.

Assume that in $\left(I_{0}\right)=$ in $(I)$. Let $f \in\left(I: x_{n}\right)$. Then $f \in I$ because otherwise the remainder $r$ of the division of $f$ by a Gröbner basis of $I$ w.r.t. the reverse lexicographic order is nonzero and in $(r) \notin$ in $(I)$. As $x_{n}$ in $(r) \in$ in $(I)$ and in $(I)=$ in $\left(I_{0}\right)$, this is impossible. Similarly, let $f \in\left(\left(I, x_{n}\right): x_{n-1}\right)$. For the same reason as above, $f \in\left(I, x_{n}\right)$ because in $\left(I, x_{n}\right)=\left(\operatorname{in}(I), x_{n}\right)$ and in $(I)=\operatorname{in}\left(I_{0}\right)$.

Conversly, if $\left\{x_{n-1}, x_{n}\right\}$ is a regular sequence on $S / I$, then the monomials in a minimal set of generators of in $(I)$ are not divisible by either $x_{n-1}$ or $x_{n}$. Thus, in $\left(I_{0}\right)=\operatorname{in}(I)$.

As already stated, $\mathfrak{C}_{0}$ is arithmetically Cohen-Macaulay by Proposition 2.1 and $\operatorname{deg} \mathfrak{C}_{0} \geq \operatorname{deg} \mathfrak{C}$. The difference between $\operatorname{deg} \mathfrak{C}_{0}$ and $\operatorname{deg} \mathfrak{C}$ is indeed a measure of how far $\mathfrak{C}$ is from being arithmetically Cohen-Macaulay.

Corollary 2.2. $\mathfrak{C}$ is arithmetically Cohen-Macaulay if and only if $\operatorname{deg} \mathfrak{C}=\operatorname{deg} \mathfrak{C}_{0}$.

Proof. The difference $\operatorname{deg} \mathfrak{C}_{0}-\operatorname{deg} \mathfrak{C}$ is equal to \#F. In fact, $\operatorname{deg} \mathfrak{C}_{0}$ is equal to $\#\left\{\alpha \in \mathbb{N}^{n-1} \mid \mathbf{x}^{(\alpha, 0,0)} \notin\right.$ in $\left.\left(I_{0}\right)\right\}$ because the Hilbert polynomial of $S / I_{0}$ is $P_{I_{0}}(T)=$ $\sum_{\alpha \notin E_{0}}(T+1-|\alpha|)$ where $E_{0}=\left\{\alpha \in \mathbb{N}^{n-1} \mid \mathbf{x}^{(\alpha, 0,0)} \in\right.$ in $\left.\left(I_{0}\right)\right\}$. By a similar argument $\operatorname{deg} \tilde{\mathfrak{C}}=\#\left\{\alpha \in \mathbb{N}^{n-1} \mid \mathbf{x}^{(\alpha, 0,0)} \notin \tilde{I}\right\}$.

Assume that $S / I$ is Cohen-Macaulay. We will give an effective method to compute reg $I$ that does not require the knowledge of a minimal graded free resolution of $I$.

Set $E:=\left\{\left(\alpha_{0}, \ldots, \alpha_{n-2}\right) \in \mathbb{N}^{n-1} \mid \mathbf{x}^{(\alpha, 0,0)} \in \operatorname{in}(I)\right\}$. As $K\left[x_{n-1}, x_{n}\right]$ is a Noether normalization of $S / I$, for $s \gg 0$ and $\alpha \in \mathbb{N}^{n-1}$ one has that $|\alpha| \geq s$ implies $\alpha \in E$. Define the regularity of $E, H(E)$, as the smallest integer $s$ satisfying this property.

Denote by $H(I)$ the regularity of the Hilbert function $H_{I}$ of $S / I$, i.e. the smallest integer $s_{0}$ such that for $s \geq s_{0}, H_{I}(s)=P_{I}(s)\left(P_{I}(T)\right.$ is the Hilbert polynomial of $S / I)$. 
Lemma 2.3. $H(E)=H(I)+2$.

Proof. As the value at $s$ of $H_{I}$ is

$$
H_{I}(s)=\#\left\{\left(\alpha_{0}, \ldots, \alpha_{n}\right) \in \mathbb{N}^{n+1} \mid \alpha_{0}+\cdots+\alpha_{n}=s \text { and }\left(\alpha_{0}, \ldots, \alpha_{n-2}\right) \notin E\right\},
$$

then $P_{I}(T)=\sum_{\alpha \notin E}(T+1-|\alpha|)$. Any element $\alpha \notin E$ satisfies $|\alpha| \leq H(E)-1$ so $H(I) \leq H(E)-1$. It is now easy to check that $H_{I}\left(s_{0}\right)=P_{I}\left(s_{0}\right)$ for $s_{0}=H(E)-2$ and that $H_{I}\left(s_{0}\right)>P_{I}\left(s_{0}\right)$ for $s_{0}=H(E)-3$.

Theorem 2.4. Let $I \subset S$ be the homogeneous defining ideal of an arithmetically Cohen-Macaulay projective curve $\mathfrak{C} \subset \mathbb{P}_{K}^{n}$. Then reg $I=H(E)$.

Proof. By the previous lemma, one has to prove that reg $I=H(I)+2$. From [6] Prop. 20.20], one gets that $\operatorname{reg} I=\operatorname{reg}\left(I, x_{n-1}, x_{n}\right)$. As $\operatorname{dim} S /\left(I, x_{n-1}, x_{n}\right)=0$, then $\operatorname{reg}\left(I, x_{n-1}, x_{n}\right)$ coincides with the regularity $H\left(I, x_{n-1}, x_{n}\right)$ of the Hilbert function of $S /\left(I, x_{n-1}, x_{n}\right)$ ([3, Lemma 1.7]). The result now follows from the equality $H\left(I, x_{n-1}, x_{n}\right)=H(I)+2$.

Example 2.5. Consider the ideal $I \subset K[x, y, z, t]$ generated by $f_{1}=x^{17} y^{14}-$ $y^{31}, f_{2}=x^{20} y^{13}, f_{3}=x^{60}-y^{36} z^{24}-x^{20} z^{20} t^{20}$. The reduced Gröbner basis of $I$ w.r.t. the reverse lexicographic order is $\left\{f_{1}, f_{2}, f_{3}, y^{48}, x^{3} y^{31}\right\}$, so in $(I)=$ $\left(x^{17} y^{14}, x^{20} y^{13}, x^{60}, y^{48}, x^{3} y^{31}\right)$. Then $K[x, y, z, t] / I$ is Cohen-Macaulay (Proposition [2.1) and reg $I=72$ (Theorem [2.4).

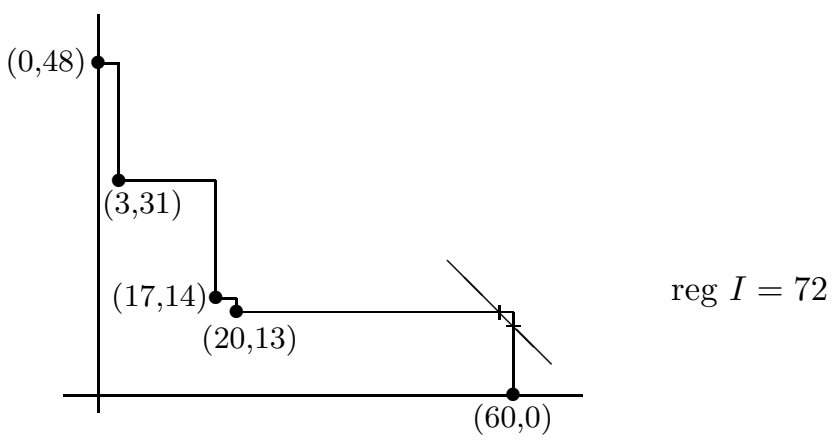

As already observed, $S / I$ is Cohen-Macaulay if and only if $S /$ in $(I)$ is CohenMacaulay. Thus, we get the following consequence of Theorem 2.4 which can also be obtained from [3 Thm. 2.4 (b)].

Corollary 2.6. If I satisfies the conditions of Theorem 2.4 then reg $I=\operatorname{reg}$ in $(I)$.

Let's assume now that $I$ is a saturated ideal defining a nonarithmetically CohenMacaulay projective curve $\mathfrak{C} \subset \mathbb{P}_{K}^{n}$. We shall give a relation between reg $I$ and reg $I_{0}$ to obtain, as in Theorem [2.4 an effective method to compute reg $I$ that does not require the knowledge of a minimal graded free resolution of $I$.

In this case $F \neq \emptyset$ (Proposition [2.1) and one has the partition introduced in [5]:

$$
\begin{aligned}
\left\{\left(\alpha_{0}, \ldots, \alpha_{n}\right) \in \mathbb{N}^{n+1} \mid\right. & \left.x_{0}^{\alpha_{0}} \cdots x_{n}^{\alpha_{n}} \notin \operatorname{in}(I)\right\}= \\
& \left\{\left(\alpha_{0}, \ldots, \alpha_{n}\right) \in \mathbb{N}^{n+1} \mid x_{0}^{\alpha_{0}} \cdots x_{n-2}^{\alpha_{n-2}} \notin \tilde{I}\right\} \cup \mathfrak{R},
\end{aligned}
$$


where $\mathfrak{R}=\bigcup_{\alpha \in F}\left\{\alpha \times\left[\mathbb{N}^{2}-E_{\alpha}\right]\right\}$ for $E_{\alpha}=\left\{\left(\alpha_{n-1}, \alpha_{n}\right) \in \mathbb{N}^{2} \mid \mathbf{x}^{\left(\alpha, \alpha_{n-1}, \alpha_{n}\right)} \in \operatorname{in}(I)\right\}$. Therefore, the value at $s \in \mathbb{N}$ of the Hilbert function $H_{I}$ of $S / I$ is

$$
H_{I}(s)=H_{\tilde{I}}(s)+\#\{\beta \in \Re|| \beta \mid=s\},
$$

where $\#\{\beta \in \mathfrak{R}|| \beta \mid=s\}$ is constant for $s \gg 0$. Denote by $H(\mathfrak{R})\left(\right.$ resp. $\left.H\left(E_{\alpha}\right)\right)$ the smallest integer $s_{0}$ such that for $s \geq s_{0}, \#\{\beta \in \mathfrak{R} \| \beta \mid=s\}\left(\right.$ resp. $\#\left\{\left(\alpha_{n-1}, \alpha_{n}\right) \in\right.$ $\left.\mathbb{N}^{2}-E_{\alpha} \mid \alpha_{n-1}+\alpha_{n}=s\right\}$ ) is constant. It is clear that

$$
H(\Re) \leq \max _{\alpha \in F}\left\{|\alpha|+H\left(E_{\alpha}\right)\right\} .
$$

Theorem 2.7. Let $I \subset S$ be a saturated ideal defining a nonarithmetically CohenMacaulay projective curve $\mathfrak{C} \subset \mathbb{P}_{K}^{n}$. Then $\operatorname{reg} I=\max \left\{\operatorname{reg} I_{0}, H(\mathfrak{R})+1\right\}$.

Proof. Since the field $K$ is infinite and $K\left[x_{n-1}, x_{n}\right]$ is a Noether normalization of $S / I$ and $I$ is a saturated ideal, then there exists $\kappa \in K-\{0\}$ such that $x_{n}-\kappa x_{n-1}$ is a nonzero divisor on $S / I$. If we denote by $I_{\kappa}$ the ideal $\left(I, x_{n}-\kappa x_{n-1}\right)$ of $S$, then $\operatorname{reg} I=\operatorname{reg} I_{\kappa}$ by [6, Prop. 20.20].

On the other hand, if $\left(I_{\kappa}\right)^{\text {sat }}$ is the saturation of $I_{\kappa}$, one deduces from [3, Lemmas 1.6, 1.7, 1.8] that reg $I_{\kappa}=\max \left\{s_{0}, H\left(I_{\kappa}, h\right)\right\}$ where $h$ is a linear form which is a nonzero divisor on $S /\left(I_{\kappa}\right)^{s a t}$, and $s_{0}$ is the smallest integer such that, for any $s \geq s_{0},\left(I_{\kappa}: h\right)_{s}=\left(I_{\kappa}\right)_{s}$.

Since $S /\left(I_{\kappa}\right)^{\text {sat }}$ is a finite $K\left[x_{n}\right]$-module of dimension 1 , then $K\left[x_{n}\right]$ is a Noether normalization of $S /\left(I_{\kappa}\right)^{\text {sat }}$ by [9, Ch. 2, Rem. 6.5.0]. Thus, $x_{n}$ is a nonzero divisor on $S /\left(I_{\kappa}\right)^{\text {sat }}$ and reg $I_{\kappa}=\max \left\{s_{0}, H\left(I_{\kappa}, x_{n}\right)\right\}, s_{0}$ being the smallest integer such that, for any $s \geq s_{0},\left(I_{\kappa}: x_{n}\right)_{s}=\left(I_{\kappa}\right)_{s}$.

Let us prove now that reg $I_{\kappa}=\max \left\{H(I)+1, H\left(I_{\kappa}, x_{n}\right)\right\}$. Indeed, as for any $s$,

$$
0 \rightarrow S_{s-1} /\left(I_{\kappa}: x_{n}\right)_{s-1} \stackrel{. x_{n}}{\longrightarrow} S_{s} /\left(I_{\kappa}\right)_{s} \stackrel{\varphi}{\longrightarrow} S_{s} /\left(I_{\kappa}, x_{n}\right)_{s} \rightarrow 0
$$

is an exact sequence, where $\varphi$ is the canonical morphism, and as $H\left(I_{\kappa}\right)=H(I)+1$, one has $\max \left\{s_{0}, H\left(I_{\kappa}, x_{n}\right)\right\}=\max \left\{H(I)+1, H\left(I_{\kappa}, x_{n}\right)\right\}$.

On the other hand, $H\left(I_{\kappa}, x_{n}\right)=\operatorname{reg} I_{0}$ because $\left(I_{\kappa}, x_{n}\right)=\left(I_{0}, x_{n-1}, x_{n}\right)$ and $I_{0}$ defines an arithmetically Cohen-Macaulay curve (see proof of Theorem 2.4).

Finally, $\max \left\{H(I)+1, \operatorname{reg} I_{0}\right\}=\max \left\{H(\Re)+1, \operatorname{reg} I_{0}\right\}$. Indeed, as in $\left(I_{0}\right) \subseteq \tilde{I}$, then $H(\tilde{I})+2=\operatorname{reg} \tilde{I} \leq \operatorname{reg} I_{0}$ by Lemma [2.3. Theorem [2.4 and Corollary [2.6], If $H(\mathfrak{R})$ and $H(I)$ are smaller or equal to $H(\tilde{I})$, the result follows from the previous inequality. Otherwise, it is easy to check that $H(\mathfrak{R})=H(I)$ and we are done.

Remark 2.8. It is worth noting that knowledge of in $(I)$ and some extra combinatorial work give the value of reg $I$. In fact, in $\left(I_{0}\right)$ is generated by the minimal generators of in $(I)$ which are not divisible by either $x_{n}$ or $x_{n-1}$ because in $\left(I_{0}\right)=$ $\theta($ in $(I)) S$. Taking $E=\left\{\alpha \in \mathbb{N}^{n-1} \mid \mathbf{x}^{(\alpha, 0,0)} \in \operatorname{in}\left(I_{0}\right)\right\}$, one gets reg $I_{0}=H(E)$ by Theorem 2.4. On the other hand, $H(\mathfrak{R})$ is also obtained from in $(I)$.

Example 2.9. For any $\ell \geq 1$, consider the saturated ideal $I_{\ell}=\left(f_{1}, f_{2}, f_{3}, h_{\ell}\right) \subset$ $K[x, y, z, t]$ generated by $f_{1}, f_{2}, f_{3}$ of the Example [2.5] and by $h_{\ell}=y^{41} z^{\ell}-y^{40} z^{\ell+1}$. One can check that $\left\{f_{1}, f_{2}, f_{3}, h_{\ell}, y^{48}, x^{3} y^{31}, y^{40} z^{\ell+8}\right\}$ is the reduced Gröbner basis of $I_{\ell}$ w.r.t. the reverse lexicographic order. Then in $\left(I_{\ell}\right)=\left(x^{17} y^{14}, x^{20} y^{13}, x^{60}\right.$, $\left.y^{41} z^{\ell}, y^{48}, x^{3} y^{31}, y^{40} z^{\ell+8}\right)$. The set $F$ is not empty and independent of $\ell$. It is 
represented by the following diagram:

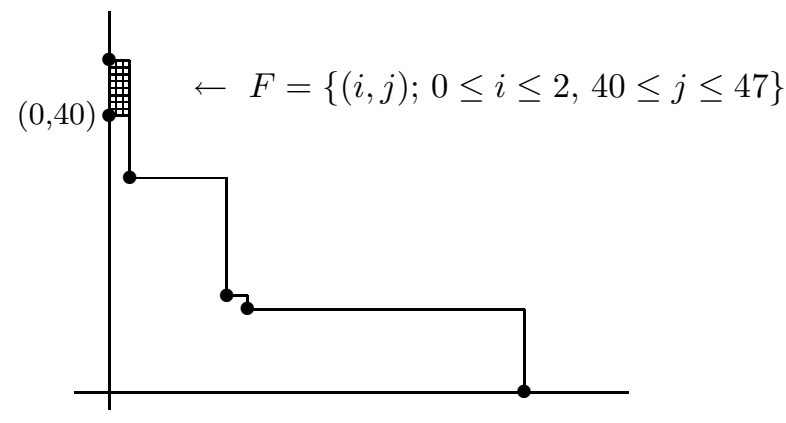

Then for $\ell \geq 1, K[x, y, z, t] / I_{\ell}$ is not Cohen-Macaulay by Proposition 2.1. Observe that for any $\ell \geq 1$, in $\left(I_{\ell}\right)_{0}$ coincides with in $(I)$, where $I$ is the ideal $\left(f_{1}, f_{2}, f_{3}\right)$ of the Example 2.5. The regularity of $\left(I_{\ell}\right)_{0}$ is then $\operatorname{reg}\left(I_{\ell}\right)_{0}=72$. Now $E_{\alpha}=(\ell+8,0)+\mathbb{N}^{2}$ for any $\alpha=(i, 40) \in F$, and $E_{\alpha}=(\ell, 0)+\mathbb{N}^{2}$ for any $\alpha=(i, j) \in F$ with $j \geq 41$. So $H(\Re)+1=\max _{\alpha \in F}\left\{|\alpha|+H\left(E_{\alpha}\right)\right\}+1=50+\ell$ and $\operatorname{reg} I_{\ell}=\max \{72,50+\ell\}$ by Theorem 2.7 .

Remark 2.10. Observe that in the previous example, in $\left(I_{\ell}\right)$ is a saturated ideal for any $\ell \geq 1$, but it is not true in general that $I=I^{\text {sat }}$ implies that in $(I)=$ in $(I)^{\text {sat }}$. For example, the ideal $I \subset K[x, y, z, t]$ generated by $x^{2}-3 x y+5 x t, x y-3 y^{2}+5 y t$, $x z-3 y z, 2 x t-y t$ and $y^{2}-y z-2 y t$ is saturated since $z-t$ is a nonzero divisor on $K[x, y, z, t] / I$ and in $(I)=\left(y z t, y^{2}, x t, x z, x y, x^{2}\right)$ is not saturated because $z-\kappa t$ is a zero divisor on $K[x, y, z, t] /$ in $(I)$, for any $\kappa \in K$. In this example, reg $I \neq \operatorname{reg}$ in $(I)$ as reg $I=2$ by Theorem $2.7\left(\operatorname{reg} I_{0}=H(\mathfrak{R})+1=2\right)$ and one can check with [4] that reg in $(I)=3$. Nevertheless, if in $(I)$ is also saturated one gets directly from Theorem 2.7 that

$$
\operatorname{reg} I=\operatorname{reg} \text { in }(I) \text {. }
$$

In particular, if $x_{n}$ is a nonzero divisor on $S / I$, one has in $(I)=$ in $(I)^{\text {sat }}$ and the above equality also comes from [3, Thm. 2.4 (b)].

The last result of this section says that the method obtained from Theorems 2.4 and 2.7 to compute the regularity of $I$ also determines when the regularity is attained at the last step of a minimal graded free resolution of $I$.

Corollary 2.11. Let $I \subset S$ be a saturated ideal defining a projective curve $\mathfrak{C} \subset \mathbb{P}_{K}^{n}$. Then reg $I$ is attained at the last step of a minimal graded free resolution of $I$ if and only if either $S / I$ is Cohen-Macaulay or $\operatorname{reg} I=H(\Re)+1$.

Proof. When $S / I$ is Cohen-Macaulay, the result is a consequence of Corollary 1.2 Assume that $S / I$ is not Cohen-Macaulay. As a consequence of the proof of Theorem 2.7, one has that $\operatorname{reg} I=H(\Re)+1$ if and only if $\operatorname{reg} I=H(I)+1$. Let

$$
0 \rightarrow \bigoplus_{j=1}^{\beta_{n-1}} S\left(-e_{n-1, j}\right) \longrightarrow \cdots \longrightarrow \bigoplus_{j=1}^{\beta_{0}} S\left(-e_{0 j}\right) \longrightarrow I \rightarrow 0
$$

be a minimal graded free resolution of $I$. The Hilbert series of $S / I$ is $\frac{Q(t)}{(1-t)^{n+1}}$ with

$$
Q(t)=1-\left(t^{e_{01}}+\cdots+t^{e_{0 \beta_{0}}}\right)+\cdots+(-1)^{n}\left(t^{e_{n-1,1}}+\cdots+t^{e_{n-1, \beta_{n-1}}}\right)
$$


and $\operatorname{deg}(Q(t))=H(I)+n$. Since $\operatorname{deg}(Q(t)) \leq \operatorname{reg} I+n-1$, and equality holds if and only if reg $I+n-1=e_{n-1}$, and the result follows.

In summary, avoiding the construction of a minimal graded free resolution of $I_{\ell}$, in Example 2.9 one can assert now that for any $\ell, 1 \leq \ell \leq 21$, the regularity of $I_{\ell}$ is attained at the second step of a minimal graded free resolution of $I_{\ell}$ but not at the third step. For $\ell \geq 22$, the regularity of $I_{\ell}$ is attained at the third step of a minimal graded free resolution of $I$ but can also occur at the second step.

\section{ACKNOWLEDGEMENTS}

We would like to thank Monique Lejeune-Jalabert and Wolmer V. Vasconcelos for helpful conversations, and Aron Simis for the corrections he suggested in a previous version of this paper.

\section{REFERENCES}

1. D. Bayer, The division algorithm and the Hilbert scheme, Thesis, Harvard University, Cambridge, MA, 1982.

2. D. Bayer and D. Mumford, What can be computed in Algebraic Geometry? In: Computational Algebraic Geometry and Commutative Algebra, Proceedings Cortona 1991 (D. Eisenbud and L. Robbiano, Eds.), Cambridge University Press, 1993, 1-48. [MR 95d:13032]

3. D. Bayer and M. Stillman, A criterion for detecting $m$-regularity, Invent. Math. 87 (1987) 1-11. MR 87k:13019

4. D. Bayer and M. Stillman, Macaulay, a system for computation in Algebraic Geometry and Commutative Algebra, 1992, available via anonymous ftp from math.harvard.edu.

5. I. Bermejo and M. Lejeune-Jalabert, Sur la compléxité du calcul des projections d'une courbe projective, to appear in Comm. in Algebra.

6. D. Eisenbud, Commutative Algebra with a view toward Algebraic Geometry, Graduate Texts in Mathematics 150, Springer, Berlin, Heidelberg, New York, 1995.

7. D. Eisenbud and S. Goto, Linear free resolutions and minimal multiplicities, J. Algebra 88 (1984) 89-133. MR 85f:13023

8. G.M. Greuel, G. Pfister and H. Schoenemann, Singular, a system for computation in Algebraic Geometry and Singularity Theory, 1995, available via anonymous ftp from helios.mathematik.uni-kl.de.

9. M. Lejeune-Jalabert, Effectivité de calculs polynomiaux, Cours de D.E.A., Institut Fourier, Grenoble, 1984-85.

10. P. Schenzel, On the use of Local Cohomology in Algebra and Geometry, In: Six Lectures on Commutative Algebra (J. Elias, J.M. Giral, R.M. Miró-Roig and S. Zarzuela, Eds.), Progress in Mathematics 166, Birkhauser, Boston, 1998.

11. W.V. Vasconcelos, Computational Methods in Commutative Algebra and Algebraic Geometry, Algorithms and Computation in Mathematics 2, Springer, Berlin, Heidelberg, New York, 1998. MR 99c:13048

Departamento de Matematica Fundamental, Facultad de Matematicas, Universidad de La Laguna, 38271-La Laguna, Tenerife, Spain

E-mail address: ibermejo@ull.es

Departamento de Algebra, Geometria y Topologia, Facultad de Ciencias, UniversiDad de Valladolid, 47005-Valladolid, Spain

E-mail address: pgimenez@wamba.cpd.uva.es 